Article

\title{
Gender-Based Differences in Actual Thumb Force Exertions at Various Target Force Levels
}

\author{
Kyeong-Hee Choi, Jae-Kyeong Kim, Hyun-Ho Shim and Yong-Ku Kong * \\ Department of Industrial Engineering, Sungkyunkwan University; Suwon, Gyeonggi-do 16419, Korea; \\ kyunghe7@naver.com (K.-H.C.); kjg9244@naver.com (J.-K.K.); shim2906@naver.com (H.-H.S.) \\ * Correspondence: ykong@skku.edu; Tel.: +82-31-290-7606
}

Received: 9 September 2019; Accepted: 23 December 2019; Published: 25 December 2019

Featured Application: This study evaluated the relationship between actual thumb-pressing force and perceived exertion levels. The results indicate a nonlinear S-shaped pattern. The study findings might be used to reduce the workload on the thumbs of employees.

\begin{abstract}
Measurement of thumb force in the industrial filed is important to quantify the risk of manual tasks. However, few studies have assessed the thumb force. Therefore, the purpose of this study was to investigate the relationship between actual thumb force and perceived force according to the force levels and gender. A total of 31 healthy participants (23 males and 8 females) were asked to exert a maximum thumb force (MTF) and random levels of target thumb force $(10,30,50,70$, and $90 \% \mathrm{MTF})$. Results indicated that females exerted approximately $73.6 \%(100.6 \mathrm{~N})$ of the strength exerted by males $(136.7 \mathrm{~N})$. Based on the differences between actual and perceived thumb exertions, the highest accuracy was achieved at 50\% MTF, designated as "50\%-phenomenon". At $10 \%$ and 30\% MTF levels, participants tended to exert greater strength (over-exertion) than the perceived exertions, whereas participants showed less strength (under-exertion) than the perceived exertions at $70 \%$ and 90\% MTFs. Participants generally exerted greater and lesser thumb force than the perceived exertions at levels lower and higher than 50\% MTF, respectively. Thus, the relationship between the actual and perceived force of exertion displayed a nonlinear S-shaped curve.
\end{abstract}

Keywords: maximum thumb force (MTF); actual thumb force exertion; perceived exertions; $50 \%$-phenomenon

\section{Introduction}

Work-related musculoskeletal disorders (WMSDs) are widespread in professions including construction, mining, manufacturing, and service industry. Although automated systems are available in automobile manufacturing, many products are still frequently handled manually. The U.S. Labor Department reported that hand and wrist pain among industry workers was the main reason for absence from work, and was closely related to productivity losses [1]. Jin et al. [2] also indicated that the right thumb and index finger were most often involved and severely injured in the study of 560 manufacturing industry workers. Rapid repetitive activities and overexertion of thumb (forceful exertions) in physiotherapy, sports, and assembly lines were reported as the leading causes of thumb injuries $[3,4]$.

Manual tasks such as initiation of fasteners and insertion of objects are frequently encountered in the automotive assembly industry and are associated with excessive thumb force ranging from $5.1 \mathrm{kgf}$ to $17.0 \mathrm{kgf}$ [5]. This level of exertion may trigger an occupational injury and represent a risk factor for WMSD. Thus, accurate measurement of thumb exertion at work and appropriate interventions, such as personal protective equipment, or modification of facilities according to the degree of thumb 
exertion, are critical to preventing occupational injury and WMSD. However, few studies have been conducted to estimate the thumb exertion among workers in the industrial sector.

Thumb force in the manufacturing field has been generally measured directly using modalities such as goniometers, force sensors, and electromyography. The thumb force signal may be collected directly using the small load cell or flexible force sensor at the skin surface of the thumb or hand tools. This method provides detailed information. However, it is more expensive and time-consuming to set up equipment and acquire data compared with indirect measurement [6]. Li and $\mathrm{Yu}$ [7] also reported that load cells are rarely used by practitioners in field studies, because of the time and effort needed for preparation and data processing. Moreover, the equipment attached to the body may interfere with workers' performance and assembly line. Thus, a direct measurement is more appropriate for laboratory assessment than the assessment of actual workplaces [6]. As mentioned above, direct measurement has limited application in workplaces entailing irregular and diverse tasks.

Indirect evaluation represents an alternative method to overcome the limitations of direct measurement. Self-reporting psychological scales are rated by workers based on their psychological perception of physical factors [8]. It is fundamentally based on the subjective evaluation of workers, and thus the results vary from person to person [6]. Borg's CR-10 [9] scale is one of the representative self-reporting methods for the estimation of subjective exertion [7]. Li and Yu [7] reported a strong linear correlation between Borg's CR-10 rating and grip force. Park and Yun [10] also estimated grip force exertion using the Borg's CR-10 scale and found that the correlation between the measured force and the perceived exertion ranged from 0.8 to 0.99 . Kong et al. [11] and Prasad and Manivannan [12] reported that the perception of handgrip force differed according to the handedness. Therefore, while substantial research has already been conducted on grip strength, very few studies investigated thumb force exertion levels and self-reporting estimates, even though the thumb plays a major role in various manual tasks [13-15].

Although several studies reported Borg's CR-10 scale as one of the representative self-reporting methods estimating subjective exertion, other studies found that the perceived exertions may vary depending on the type of muscle. Frank et al. [16] investigated the relationship between perceived exertion and action force exertions in maintaining the same force levels ( $20 \%$ maximum voluntary contraction (MVC)) on the legs and arms for five minutes of exercise time. The results showed significant differences in perceived exertion levels depending on the type of muscle: the RPE (Rating of Perceived Exertion) in the legs was greater than the force of the arm). The Borg's CR-10 scale was developed based on \% MVC and multiplied by 10 on the Borg CR-10 scale to obtain \%MVC (i.e., 5 on the Borg CR-10 scale corresponds to 50\% MVC).

Thus, \% MVC was used instead of Borg CR-10 scales as a measure of subjectively perceived exertions in this study to elucidate the relationship between perceived and actual thumb force exertion levels.

If there is a strong correlation between actual thumb force exertion and perceived force of thumb exertion, it is easy to estimate the thumb force in industrial fields based on the subjective perception of thumb force. Therefore, the objective of the current study was to investigate the relationship between actual thumb force exertion and perceived thumb force exertion according to the various levels of exertions (10, 30, 50, 70, and 90\% maximum thumb force (MTFs)) and genders.

Previous studies that evaluated perceived ratings of grip strength indicated a nonlinear relationship between the perceived and actual force of exertion [11]. Pinchvero et al. [17,18] also reported a nonlinear relationship between the perceived and actual force of exertion. Therefore, the hypothesis for this study was that it might be a non-linear relationship between actual and perceived thumb force exertion. In addition, the non-linear relationship might differ between genders. 


\section{Methods}

\subsection{Participants}

A total of 31 participants (23 males and eight females) were recruited through advertising at Sungkyunkwan University. All participants were right-handed and had no history of musculoskeletal disorders related to the upper limbs, fingers, or thumbs. The average and standard deviations of all the participants' height, weight, hand length, thumb length, hand width, and hand depth are listed in Table 1.

Table 1. Participants' anthropometric data (Mean \pm SD).

\begin{tabular}{ccc}
\hline \multirow{2}{*}{ Anthropometry Item } & \multicolumn{2}{c}{ Gender } \\
\cline { 2 - 3 } & Male & Female \\
\hline Age (year) & $22.9 \pm 1.2$ & $21.8 \pm 1.2$ \\
Height $(\mathrm{cm})$ & $175.2 \pm 5.5$ & $161.2 \pm 3.4$ \\
Weight $(\mathrm{kg})$ & $73.7 \pm 11.6$ & $51.2 \pm 5.3$ \\
Hand length $(\mathrm{mm})$ & $185.4 \pm 6.8$ & $168.5 \pm 4.8$ \\
Thumb length $(\mathrm{mm})$ & $63.1 \pm 3.5$ & $56.9 \pm 4.4$ \\
Hand width $(\mathrm{mm})$ & $81.9 \pm 3.5$ & $73.6 \pm 3.2$ \\
Hand depth $(\mathrm{mm})$ & $29.7 \pm 2.8$ & $24.9 \pm 2.6$ \\
\hline
\end{tabular}

SD denotes standard deviation.

\subsection{Apparatus}

A sub-miniature load cell (Honeywell Model 13, Sensotec, Columbus, OH, USA ranging from 0-50 kg with a $9.5 \mathrm{~mm}$ diameter and 2.75 thickness) was used to measure the maximum thumb force (MTF) and sub-maximum thumb forces (Figure 1). Calibration of the load cell was performed using known weights $(1-5 \mathrm{~kg})$ on a custom-made fixture. The load cell showed high linearity between the output data (millivolts per volt, $\mathrm{mV} / \mathrm{V})$ and the applied known weight $\left(\mathrm{R}^{2}>0.99\right)$. The output data were collected using an NI-USB-6259 DAQ board and presented on the screen using a custom-made software program written in LabVIEW (National Instruments, Austin, TX, USA). This software was programmed to convert the output data of the load cell $(\mathrm{mV} / \mathrm{V})$ into force value $(\mathrm{N})$ and to measure the data in real-time. For visual information, the thumb force data are represented by a blue line on the screen in real-time (Figure 2, right). When participants exerted their thumb forces for $5 \mathrm{~s}$ (Figure 2, left), the data were collected and restored at a sampling rate of $100 \mathrm{~Hz}$.
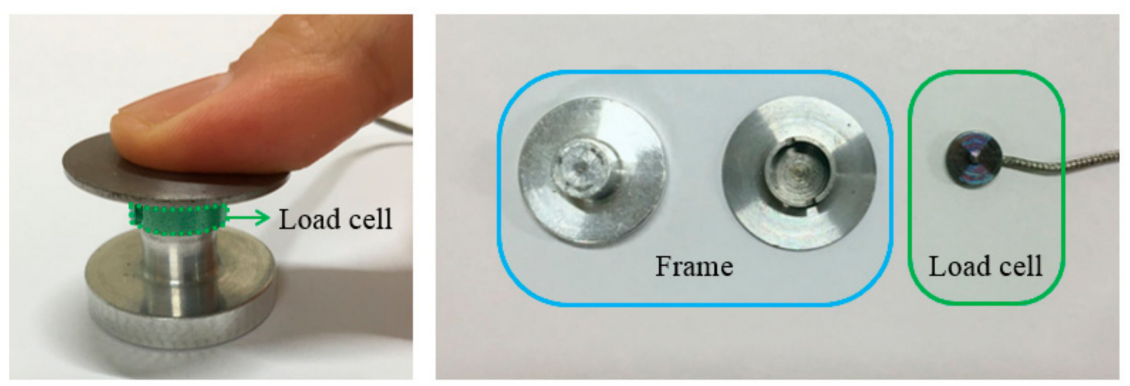

Figure 1. Hardware of thumb force measurement system. 

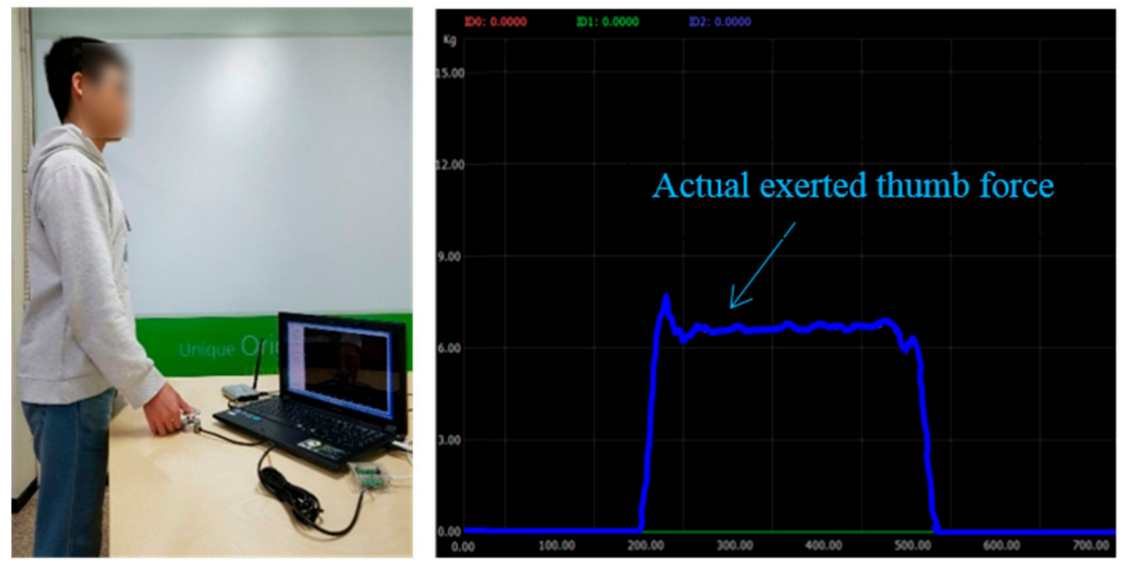

Figure 2. Experimental posture (left) and thumb force exertion measurement program (right).

\subsection{Experimental Procedure}

A brief description of the objectives and procedures was provided to each participant, and the hand and thumb lengths, hand width and depth were measured using a digital caliper (Mitutoyo 500-182-20, Mitutoyo Corp., Kawasaki, Kanagawa, Japan). Participants were asked to maintain a standing posture and to exert their MTF on the load cell for $5 \mathrm{~s}[19,20]$. The MTF was exerted three times with a 3-min resting time between the trials. The investigator calculated the average of all the three maximum thumb forces of each participant and set an individual MTF for each participant. The investigator randomly selected a perceived exertion level (\% MTF), which was one of the five levels of $\%$ MTF (10-90\% MTF at an interval of $20 \%$ ) with two repetitions. Thus, all the participants were asked to exert 10 thumb force exertions for the perceived level of exertion (\% MTF), which was selected according to the investigator's judgment for $5 \mathrm{~s}$ (Figure 2).

\subsection{Experimental Design}

The independent variables included two levels of gender (male and female) and five levels of perceived exertion $(10 \%, 30 \%, 50 \%, 70 \%$, and $90 \% \mathrm{MTFs})$ for the determination of actual exerted thumb force, and the difference between actual and perceived exertions. The MTF was the only independent gender variable. Tukey tests followed ANOVA with SPSS 18.0 (Lead Technologies, Inc., Chicago, IL, USA).

A 2 (gender) $\times 5(\% \mathrm{MTF})$ within-subject design was used for the analysis of variance (ANOVA) with a significance level of 0.05 . The difference between actual and target thumb force was calculated by Equation (1).

$$
\operatorname{dTF}(\%)=\left[\frac{a T F-t T F}{t T F}\right] \times 100
$$

In the Equation (1) above, dTF (\%) is the percentage difference between actual thumb force and target thumb force, tTF denotes the target thumb force (\% MTF) calculated based on the MTF for each participant and randomly selected by the investigator, and $a T F$ represents the actual thumb force exerted by each participant for the target thumb force $(t T F)$.

Polynomial regression analysis was used to predict the actual exerted force from the perceived exertion force for males and females, as shown in Equation (2), where $y_{a c}$ represents the actual exerted force, $\beta_{j}$ represents the regression coefficient, and $x_{p e r}$ is the perceived thumb force.

$$
y_{a c}=\beta_{0}+\beta_{1} x_{p e r}+\beta_{2} x_{p e r}^{2}+\beta_{3} x_{p e r}^{3}+\cdots+\beta_{d} x_{p e r}^{d}
$$




\section{Results}

\subsection{Maximum Thumb Force (MTF)}

Statistical analysis of the MTF suggested that the main effect of the gender was statistically significant in this study $(p<0.05)$. The average MTF of females was $100.62 \mathrm{~N}$, which corresponds to $73.62 \%$ of the MTF $(136.71 \mathrm{~N})$ of males.

\subsection{Actual Exerted Thumb Force}

The results of ANOVA showed that the actual exerted thumb force varied significantly according to the perceived exertion levels ( $p<0.05$, Figure 3 ), whereas the effects of gender and the interaction between gender and perceived exertion levels were not significant. As expected, the Tukey test showed that participants actually exerted the largest thumb force (79.2 $\pm 15.9 \% \mathrm{MTF})$ at 90\% MTF perceived level of exertion followed by $49.4 \pm 15.2 \%$ and $58.8 \pm 16.8 \%$ MTFs at $50 \%$ and $70 \%$ MTF perceived exertion level, respectively. The $38.2 \pm 13.8 \%$ MTF was also obtained at $30 \%$ MTF perceived exertion level, and the least actual thumb force $(21.7 \pm 10.2 \% \mathrm{MTF})$ was obtained at $10 \%$ MTF perceived exertion level in both genders. There was no statistically significant difference between the actual thumb forces exerted at 50\% perceived exertion level in this study (Figure 3, Table 2).

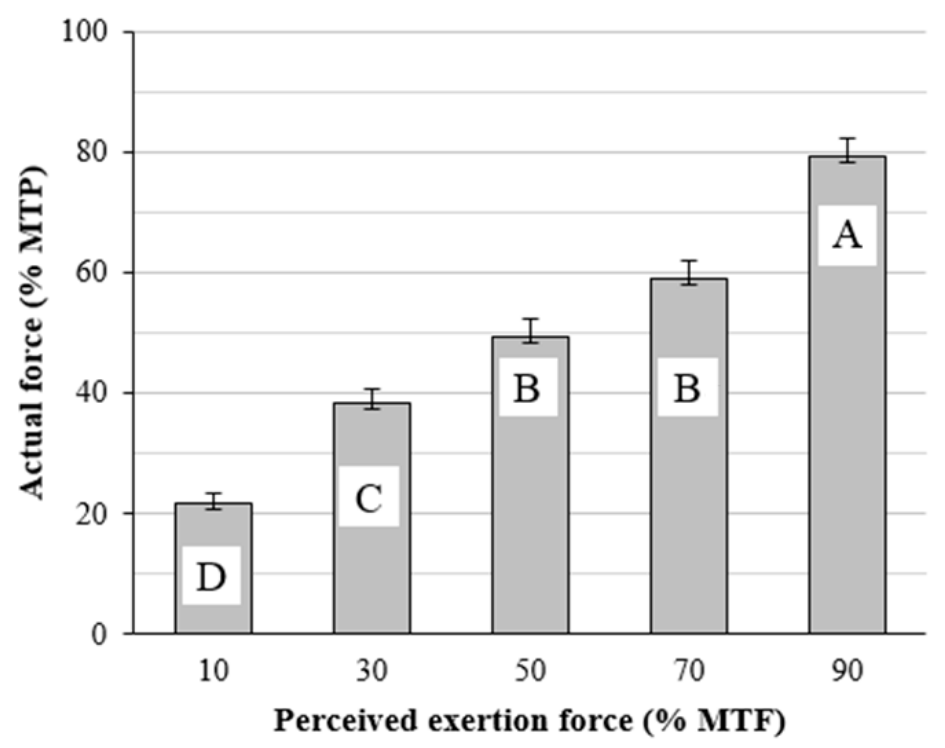

Figure 3. Actual exerted \% MTF according to perceived exertion level (\% MTFs). MTF denotes maximum thumb force. Alphabetic letters denote statistically significant groupings, $p=0.05$.

Table 2. Comparison between actual and perceived exertion level (\% MTFs).

\begin{tabular}{cccc}
\hline Perceived Exertion Force (\% MTF) & $\begin{array}{c}\text { Actual Exerted Force } \\
\text { (Mean } \pm \text { SD, \% MVC) }\end{array}$ & $\mathbf{t}_{\mathbf{2 9}}$ & $\boldsymbol{p}$-Value \\
\hline $10^{*}$ & $21.7 \pm 10.2$ & 6.109 & $<0.001$ \\
$30 *$ & $38.2 \pm 13.8$ & 3.310 & 0.002 \\
50 & $49.4 \pm 15.2$ & -0.206 & 0.838 \\
$70 *$ & $58.8 \pm 16.8$ & -3.700 & 0.001 \\
$90 *$ & $79.2 \pm 15.9$ & -3.780 & 0.001 \\
\hline
\end{tabular}

MTF denotes maximum thumb force. MVC denotes maximum voluntary contraction. SD denotes standard deviation. * indicates that the actual exerted force was significantly different from the perceived exertion $(p<0.05)$.

$t$-test was conducted to determine statistically significant differences between perceived and actual exertion. As shown in Table 2, a significant difference existed between perceived and actual forces of exertion except for the 50\% MTF. In general, participants exerted greater actual thumb force than the 
perceived exertion level under the $50 \% \operatorname{MTF}(10,30 \% \mathrm{MTF})$, whereas less actual thumb force than the perceived exertion above $50 \%$ MFT (70, 90\% MTFs).

\subsection{Difference between Actual Thumb Force and Perceived Exertion Level (\% MTF)}

The difference between actual thumb force exerted and the perceived exertion level was primarily affected by the perceived exertion levels $(p<0.05)$. The actual thumb force showed greater exertions $(117 \%$ and $27 \%$ ) than the perceived exertion levels (10 and 30\% MTFs), whereas fewer exertions $(-16 \%$ and $-12 \%)$ than the perceived exertion levels (70 and $90 \%$ MTFs), respectively. Notably, most participants accurately exerted actual thumb forces for $50 \%$ of the MTF level in this study (Figure 4).

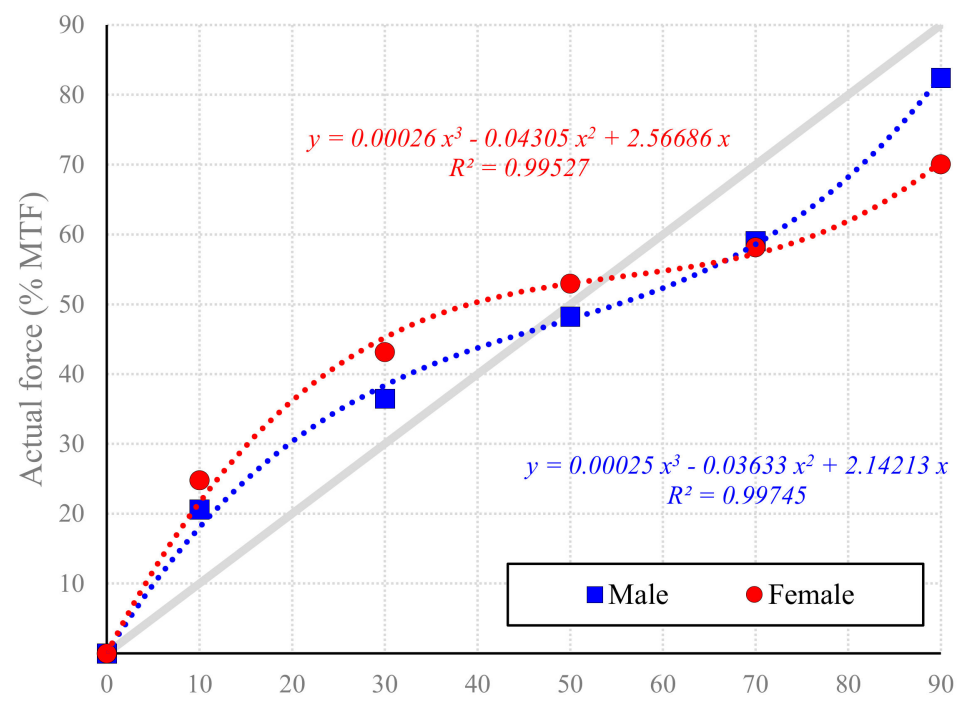

Figure 4. Interaction effect of gender and perceived exertion levels (\%MTF).

Although the interaction between gender and target force level of perceived exertion was not statistically significant, generally females exerted higher thumb forces than males at $0-50 \%$ MTF target force and less thumb force than males at 50-90\% MTF target force level of perceived exertion (Figure 4).

To predict the actual thumb force, a polynomial regression of the order 3 model was used (Figure 4). The proposed models are represented by the following Equations (3) and (4) for males and females, respectively, where $y_{a c}$ is the predicted value of actual thumb force and $x_{p e r}$ is the perceived thumb force. Both models showed an R-squared value greater than 0.99 .

$$
\begin{aligned}
& y_{a c}=0.00025 x_{p e r}^{3}-0.03633 x_{p e r}^{2}+2.14213 x_{p e r} \\
& y_{a c}=0.00026 x_{p e r}^{3}-0.04305 x_{p e r}^{2}+2.56686 x_{p e r}
\end{aligned}
$$

\section{Discussion}

This study was conducted to investigate the relationship between actual thumb force exertion and perceived thumb force exertion using the Borg's CR-10 scale. The relationship between actual and perceived thumb force exertions shows a non-linear S-shape curve. Most participants exerted their actual thumb force at 50\% MTF accurately and with high accuracy. Based on the $50 \%$ MTF level, over-exertion was observed at 50\% MTF or below, and under-exertion was found at $50 \%$ MTF level or above, and therefore, designated as the ' $50 \%$ phenomenon'.

Several previous studies reported that the maximum force exerted by males was greater than that of females in pinching, grasping, and lifting tasks [21,22]. In studies of elbow flexion and grip strength, females exerted $39.5-45.0 \%$ [23,24] and 52.9-62.3\% [25-28] of male strength, respectively. The thumb force exertion studies [29-31] also showed that females exerted lower thumb forces (64.1-97.1\%) than 
males. The current study was similar to that of previous studies involving thumb force exertions (female MTF was about $73.6 \%$ that of males).

The difference between males and females in terms of maximum muscle exertions might be attributed to the size of physiological cross-sectional areas (PCSA) of muscle groups [32,33].

The results of this study demonstrated that males exerted significantly greater thumb force (absolute magnitude) than females across all perceived exertion levels. However, when normalized to \% MTF for each participant's MTF, there was no difference between male and female exertion as a function of the perceived exertion level (Figure 3). Pincivero et al. [17,34] also compared the perceived exertion of quadriceps femoris muscles with target forces at 10-90\% MVCs. They found no significant difference between genders, although males generally lift or exert masses or forces of absolutely greater magnitude than females.

The differences in actual exertion of different levels of perceived exertion were also investigated in this study. Results showed that the exertion force of \% MTF differed significantly $(p<0.05)$ at the perceived exertion levels of 10, 30, 70, and 90\% MTFs, except at 50\% MTF. The actual exertions of $10 \%$ and $30 \%$ MTFs were greater (over-exertion) than the perceived exertion levels, whereas the exertions for $70 \%$ and $90 \%$ MTFs were less (under-exertion) than that of the perceived exertion. Although the interaction between gender and perceived exertion on each \% MTF was not statistically significant, females generally showed over-exertion at 10-30\% MTF and under-exertion at 70-90\% levels, when compared with males. Notably, they accurately exerted thumb forces at $50 \%$ MTF level in both genders (around 1.2\% difference, Figure 3).

Thus, the relationship between perceived thumb force exertion (\% MTF) and actual thumb force (\% MTF) showed an S-shaped pattern (Figure 4). It suggested a tendency to exert higher and lower thumb forces than the perceived levels of exertion at lower and higher than 50\% MTF levels, respectively, as shown in Figure 4. Therefore, 50\% MTF is an inflection point where curves change from concave to convex, suggesting that the participants were biased toward over-exertion under the $50 \%$ MTF level, and under-exertion above the 50\% MTF level, respectively. It represents a "50\%-phenomenon". This finding was also similar to that of previous studies, which reported that the actual forces of exertion trended toward over-exertion at low levels of perceived exertion, whereas the actual forces of exertion presented a trend toward under-exertion at high levels of perceived exertion involving pinching, gripping, lifting, and leg extension tasks among both genders [35-38].

The findings of this study may be useful in assessing the workload of workers using thumb exertion forces in the manufacturing process. As noted earlier in the introduction, direct measurement of load cells is accurate, but is expensive and time-consuming, and therefore, not suitable for the actual work sites. Based on the regression equation derived from our research, the workers' thumb exertions were measured easily and accurately (both regression models with an $\mathrm{R}^{2}$ of more than 0.99 ). In addition, our research findings may contribute to the prevention of WMSDs at work sites. Accurate measurement of risk factors is essential for interventions to improve the manufacturing line. Therefore, it is necessary to develop effective measures to assess the risk factors for thumb-related WMSDs.

There are several limitations to this study. First, our study only targeted participants in their 20s and may not represent the working population in the manufacturing industry. Therefore, further studies involving various participants differing in age, gender, industry, and anthropometric characteristics are needed to address the limitations of this study. The effect of other limiting factors such as weight, height, and handgrip strength was not evaluated. In order to overcome these limitations, additional studies incorporating these variables should be considered. Although this study has some limitations, the authors expect that the results of this study could be used as fundamental data to understand the relationship between actual and perceived exertion of thumb force.

\section{Conclusions}

MTF and the difference between actual and perceived forces of exertion $(10,30,50,70$ and $90 \%$ MTFs) were evaluated in this study. 
Results showed that the MTF of females was about $73.62 \%$ that of males. In the differential analyses of actual and perceived exertion levels, a very high accuracy (1.2\% differences) was obtained at 50\% MTF, suggesting a "50\%-phenomenon". In addition, over-and under-exertions were detected at levels lower than 50\% MTF and at levels higher than 50\% MTF, respectively, yielding an S-shaped non-linear graph.

The results of this study may facilitate the estimation of the thumb force in the manufacturing field. The study data might also be used as a guideline for thumb pressing tasks in industries such as automobile assembly lines.

Author Contributions: Investigation, K.-H.C.; Methodology, H.-H.S.; Project administration, Y.-K.K.; Software, J.-K.K. All authors have read and agreed to the published version of the manuscript.

Funding: This study was carried out with the support of the "Cooperative Research Program for Agriculture Science and Technology Development (Project No. PJ012509032019)" Rural Development Administration, Republic of Korea.

Conflicts of Interest: The authors declare no conflict of interest.

\section{References}

1. United States Department of Labor. Lost-Work Time Injuries and Illness: Characteristics and Resulting Days away from Work; Bureau of Labor Statistics: Washington, DC, USA, 2005.

2. Jin, K.; Lombardi, D.; Courtney, T.; Sorock, G.; Li, M.; Pan, R.; Wang, X.; Lin, J.; Liang, Y.; Perry, M. Patterns of work-related traumatic hand injury among hospitalised workers in the People's Republic of China. Inj. Prev. 2010, 16, 42-49. [CrossRef]

3. Kim, B. Wrist and hand pain. J. Korean Acad. Fam. Med. 2000, 21, 820-836.

4. Eerkes, K. Volleyball injuries. Curr. Sports Med. Rep. 2012, 11, 251-256. [CrossRef] [PubMed]

5. Kong, Y.-K.; Lee, J.-H.; Lee, J.-H.; Shin, J.-M.; Park, C.W.; Kim, B.C.; Choi, K.-H. The Development of Guideline for Risk Evaluation of Thumb-Pressing Tasks Associated with Thumb-Pressing Forces and Repetitions. J. Korean Inst. Ind. Eng. 2018, 44, 47-53.

6. Wang, D.; Dai, F.; Ning, X. Risk Assessment of Work-Related Musculoskeletal Disorders in Construction: State-of-the-Art Review. J. Constr. Eng. Manag. 2015, 141, 04015008. [CrossRef]

7. Li, K.W.; Yu, R. Assessment of grip force and subjective hand force exertion under handedness and postural conditions. Appl. Ergon. 2011, 42, 929-933. [CrossRef]

8. Spielholz, P. Calibrating Borg scale ratings of hand force exertion. Appl. Ergon. 2006, 37, 615-618. [CrossRef]

9. Borg, G. Psychophysical scaling with applications in physical work and the perception of exertion. Scand. J. Work. Environ. Heal. 1990, 16, 55-58. [CrossRef]

10. Park, D.; Yun, M.H. An application of psychophysical ratings to external force estimation. Comput. Ind. Eng. 1996, 31, 675-680. [CrossRef]

11. Kong, Y.-K.; Lee, J.-H.; Shin, J.-M.; Shim, H.-H.; Kim, J.-K.; Cho, M.-U.; Park, C.-W.; Choi, K.-H. Evaluation of subjective perceived rating for grip strength depending on handedness for various target force levels. Work 2019, 62, 21-26. [CrossRef]

12. Manivannan, M.; Prasad, M.R. Comparison of Force Matching Performance in Conventional and Laparoscopic Force-Based Task. Proc. Hum. Factors Ergon. Soc. Annu. Meet. 2014, 58, 683-687.

13. Cotugno, G.; Althoefer, K.; Nanayakkara, T. The role of the thumb: Study of finger motion in grasping and reachability space in human and robotic hands. IEEE Trans. Syst. Man. Cybern. Syst. 2017, 47, 1061-1070. [CrossRef]

14. Feix, T.; Romero, J.; Schmiedmayer, H.-B.; Dollar, A.M.; Kragic, D. The GRASP Taxonomy of Human Grasp Types. IEEE Trans. Hum.-Mach. Syst. 2016, 46, 66-77. [CrossRef]

15. Curran, P.F.; Bagley, A.M.; Sison-Williamson, M.; James, M.A. Three-dimensional functional workspace of thumb prehension. Clin. Biomech. 2019, 63, 63-72. [CrossRef]

16. Franke, W.D.; Boettger, C.F.; McLean, S.P. Effects of varying central command and muscle mass on the cardiovascular responses to isometric exercise. Clin. Physiol. 2000, 20, 380-387. [CrossRef]

17. Pincivero, D.M.; Coelho, A.J.; Erikson, W.H. Perceived exertion during isometric quadriceps contraction. A comparison between men and women. J. Sports Med. Phys. Fit. 2000, 40, 319-326. 
18. Pincivero, D.M.; Coelho, A.J.; Campy, R.M. Gender Differences in Perceived Exertion during Fatiguing Knee Extensions. Med. Sci. Sports Exerc. 2004, 36, 109-117. [CrossRef]

19. Stackhouse, S.K.; Stevens, J.E.; Lee, S.C.; Pearce, K.M.; Snyder-Mackler, L.; Binder-Macleod, S.A. Maximum voluntary activation in nonfatigued and fatigued muscle of young and elderly individuals. Phys. Ther. 2001, 81, 1102-1109.

20. Boettcher, C.E.; Ginn, K.A.; Cathers, I. Standard maximum isometric voluntary contraction tests for normalizing shoulder muscle EMG. J. Orthop. Res. 2008, 26, 1591-1597. [CrossRef]

21. Pheasant, S. Sex differences in strength-Some observations on their variability. Appl. Ergon. 1983, 14, 205-211. [CrossRef]

22. Härkönen, R.; Piirtomaa, M.; Alaranta, H. Grip Strength and Hand Position of the Dynamometer in 204 Finnish Adults. J. Hand Surg. 1993, 18, 129-132. [CrossRef]

23. Hunter, S.K.; Enoka, R.M. Sex differences in the fatigability of arm muscles depends on absolute force during isometric contractions. J. Appl. Physiol. 2001, 91, 2686-2694. [CrossRef] [PubMed]

24. Yoon, H.; Kim, E. Muscle strength measurement using shoulder and upper joint for Korean young-aged. J. Ergon. Soc. Korean 2009, 28, 125-134.

25. Talsania, J.S.; Kozin, S.H. Normal digital contribution to grip strength assessed by a computerized digital dynamometer. J. Hand Surg. 1998, 23, 162-166. [CrossRef]

26. Kong, Y.-K.; Lowe, B.D. Optimal cylindrical handle diameter for grip force tasks. Int. J. Ind. Ergon. 2005, 35, 495-507. [CrossRef]

27. Kong, Y.-K.; Sohn, S.-T.; Kim, D.-M.; Jung, M.-C. Grip force, finger force, and comfort analyses of young and old people by hand tool handle shapes. J. Ergon. Soc. Korea 2009, 28, 27-34.

28. Kong, Y.-K.; Kim, D.-M.; Park, J.-S.; Lee, S.-Y.; Choi, K.-H.; Kim, K.R. Evaluation of Gender Effect in Various Pliers' Grip Spans for Maximum Isometric Grasping Tasks. J. Ergon. Soc. Korea 2014, 33, 553-563. [CrossRef]

29. Fulco, C.S.; Rock, P.B.; Muza, S.R.; Lammi, E.; Cymerman, A.; Butterfield, G.; Moore, L.G.; Braun, B.; Lewis, S.F. Slower fatigue and faster recovery of the adductor pollicis muscle in women matched for strength with men. Acta Physiol. Scand. 1999, 167, 233-239. [CrossRef]

30. Ditor, D.S.; Hicks, A.L. The effect of age and gender on the relative fatigability of the human adductor pollicis muscle. Can. J. Physiol. Pharmacol. 2000, 78, 781-790. [CrossRef]

31. Lee, J.-H.; Kong, Y.-K.; Choi, K.-H.; Lee, S.-Y. Development of thumb endurance curves associated with various exertion levels. Hum. Factors Ergon. Manuf. 2017, 27, 249-255.

32. Bruce, S.A.; Newton, D.; Woledge, R.C. Effect of age on voluntary force and cross-sectional area of human adductor pollicis muscle. Q. J. Exp. Physiol. 1989, 74, 359-362. [CrossRef] [PubMed]

33. Miller, A.E.J.; MacDougall, J.D.; Tarnopolsky, M.A.; Sale, D.G. Gender differences in strength and muscle fiber characteristics. Graefe's Arch. Clin. Exp. Ophthalmol. 1993, 66, 254-262. [CrossRef] [PubMed]

34. Pincivero, D.M.; Coelho, A.J.; Campy, R.M.; Salfetnikov, Y.; Bright, A. The effects of voluntary contraction intensity and gender on perceived exertion during isokinetic quadriceps exercise. Graefe's Arch. Clin. Exp. Ophthalmol. 2001, 84, 221-226. [CrossRef] [PubMed]

35. Kumar, S.; Simmonds, M.; Lechelt, D. Maximal and graded effort perception by young females in stoop lifting, hand grip and finger pinch activity with comparisons to males. Int. J. Ind. Ergon. 1994, 13, 3-13. [CrossRef]

36. Kumar, S.; Narayan, Y.; Chouinard, K. Effort reproduction accuracy in pinching, gripping, and lifting among industrial males. Int. J. Ind. Ergon. 1997, 20, 109-119. [CrossRef]

37. Kumar, S.; Simmonds, M. The accuracy of magnitude production of submaximal precision and power grips and gross motor efforts. Ergonomics 1994, 37, 1345-1353. [CrossRef]

38. Jackson, A.W.; Ludtke, A.W.; Martin, S.B.; Koziris, L.P.; Dishman, R.K. Perceived Submaximal Force Production in Young Adults. Res. Q. Exerc. Sport 2006, 77, 50-57. [CrossRef]

(C) 2019 by the authors. Licensee MDPI, Basel, Switzerland. This article is an open access article distributed under the terms and conditions of the Creative Commons Attribution (CC BY) license (http://creativecommons.org/licenses/by/4.0/). 